\title{
Índice de qualidade de água modificado pela análise multivariada: estudo de caso do Arroio Pelotas, RS, Brasil
}

\author{
Water quality index modified by using multivariate \\ analysis: a case study of Pelotas Stream, RS, Brazil
}

Samanta Tolentino Cecconello'*, Luana Nunes Centeno', Hugo Alexandre Soares Guedes'

口-

\begin{abstract}
RESUMO
O objetivo deste estudo foi desenvolver um índice de qualidade de água modificado $\left(\mathrm{IQA}_{\mathrm{Mod}}\right)$, por meio de análises multivariadas, e comparar com o índice utilizado pela Companhia Ambiental do Estado de São Paulo (Cetesb) $\left({ }^{Q} A_{\text {CETESB }}\right)$, para ser aplicado no Arroio Pelotas, Rio Grande do Sul, Brasil. Foram usados dados secundários de qualidade da água superficial do Arroio Pelotas, para o sítio de amostragem GER 48, localizado no município de Pelotas, monitorados e disponibilizados pela Fundação Estadual de Proteção Ambiental Henrique Luís Roessler (FEPAM), no período entre 1997 e 2013. Entre as análises multivariadas, empregou-se a análise fatorial (AF)/análise de componentes principais (ACP). Os resultados indicaram que a ACP extraiu duas componentes principais que explicaram 100\% da variância total dos dados na matriz de correlação, enquanto os escores fatoriais para o cálculo do IQA $\mathrm{Mod}_{\text {foram }}$ extraídos do primeiro fator, que explicou 70,6\% da variância total. Concluímos

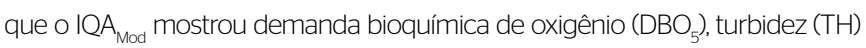
e sólidos totais (ST) como as variáveis de mais importância na construção do índice, diferindo do IQA CETESB' que apresentou as variáveis oxigênio dissolvido (OD), coliformes termotolerantes (CT) e potencial hidrogeniônico $(\mathrm{pH})$ como as de mais relevância, demonstrando assim a necessidade da calibração do IQA $A_{\text {CETESB }}$ para aplicação nesse ecossistema especííco.
\end{abstract}

Palavras-chave: qualidade da água; índice de qualidade de água; análise multivariada; Arroio Pelotas.

\begin{abstract}
This research aimed at developing a modified water quality index (WQI ${ }_{\text {Mod }}$ ) by using multivariate analysis, from a comparison with the WQI used by the Environmental Company of São Paulo (Companhia Ambiental do Estado de São Paulo-Cetesb) (IQA CETeSB $_{\text {, }}$, to be applied in the Pelotas Stream, RS, Brazil. Secondary data from Pelotas Stream surface water quality were used, collected in the sampling site GER 48, located in the municipality of Pelotas, where they were monitored and provided by the State Foundation of Environmental Protection Henrique Luís Roessler (Fundação Estadual de Proteção Ambiental Henrique Luís Roessler-FEPAM) between 1997 and 2013. Among the multivariate analyses, the factor analysis/principal component analysis (PCA) was used. The results indicated that the PCA extracted two principal components, which explained $100 \%$ of the total data variance in the correlation matrix, while the factorial scores for $\mathrm{WQI}_{\text {Mod }}$ calculation were extracted from the first factor, which explained $70.6 \%$ of the total data variance. We concluded that the WQI ${ }_{\text {Mod }}$ exhibits biochemical oxygen demand $\left(\mathrm{BOD}_{5}\right)$, turbidity $(\mathrm{TH})$ and total solids (TS) as the most important variables for the index construction, differing from the WQI ${ }_{\text {CETESB, }}$ which shows the variables dissolved oxygen (DO), thermotolerant coliforms (TC) and hydrogenionic potential $(\mathrm{pH})$ as the most relevant ones, indicating the need of WQI $I_{\text {CETESB }}$ calibration for applying in this specific ecosystem.
\end{abstract}

Keywords: water quality; water quality index; multivariate analysis; Pelotas stream.

\section{INTRODUÇÃO}

A qualidade da água está intimamente ligada às suas características físicas, químicas e biológicas, e sua condição é função dos diferentes usos a que se destina (ABREU \& CUNHA, 2015). As modificações que ocorrem nas características da bacia hidrográfica e que de alguma forma alteram o equilíbrio e a dinâmica dos corpos d'água estão associadas à poluição existente no território (POLETO, 2014).
Uma alternativa muito utilizada em estudos ambientais para analisar as relações que ocorrem nos corpos hídricos é a técnica estatística multivariada, que, de acordo com Hair et al. (2009), analisa simultaneamente múltiplas medidas sobre indivíduos ou objetos de investigação.

Segundo Olsen, Chappell e Loftis (2012), os métodos estatísticos multivariados podem ser empregados em dados coletados ao longo do tempo e em vários locais dentro de uma bacia hidrográfica para 
melhor compreender as relações entre os parâmetros monitorados. Para Ouyang (2005), um dos métodos mais utilizados na estatística multivariada, para geração de fatores, aplicada em estudos ambientais, é a análise fatorial. Já para a extração desses fatores é comumente empregada a análise de componentes principais (ACP) (AL-MUTAIRI; ABAHUSSAIN; EL-BATTAY, 2014; BERTOSSI et al., 2013; GOMES et al., 2014; MORETTO et al., 2012; OUYANG, 2005; SIMEONOV et al., 2003; SUN et al., 2016).

Além do uso da estatística multivariada, é possível utilizar índices de qualidade ambiental cuja finalidade é servir como instrumento de gestão ambiental e comunicação para a população em geral sobre as condições ambientais dos corpos d'água (SPERLING, 2005). Existem vários índices de qualidade ambiental, com diversas finalidades e objetivos, como o índice de qualidade da água (IQA), o índice de toxicidade (IT), o índice de estado trófico (IET), o índice de diversidade de espécies, entre outros (LIBÂNIO, 2010).

O IQA vem sendo utilizado como ferramenta para o monitoramento da qualidade da água, pois permite, por intermédio de informações resumidas, prever as condições do manancial superficial ao longo do tempo, reduzir custos e tempo e fornecer resultados de fácil interpretação (SILVEIRA et al., 2014; CUNHA et al., 2013).

O IQA foi proposto pela National Sanitation Foundation (NSF), nos Estados Unidos, no ano de 1970. No Brasil, ele vem sendo utilizado pela Companhia Ambiental do Estado de São Paulo (CETESB), pelo Comitê de Gerenciamento da Bacia Hidrográfica do Rio dos Sinos (COMITESINOS) e pela Fundação Estadual de Proteção Ambiental Henrique Luís Roessler (FEPAM), entre outros órgãos que também realizaram adaptações no IQA e passaram a usá-lo como ferramenta de monitoramento ambiental e suporte à tomada de decisão (BLUME et al., 2010; SUN et al., 2016). A modificação do IQA utilizado pela CETESB (IQA ${ }_{\text {CETESB }}$ ) faz-se necessária, porque o índice modificado leva em consideração as características próprias de cada manancial (MORETTO et al., 2012). Dessa forma, cada variável que compõe o índice receberá um peso de acordo com seu grau de importância (SUN et al., 2016).

Diante disso, os objetivos deste estudo foram desenvolver um IQA modificado $\left(\mathrm{IQA}_{\mathrm{Mod}}\right)$ por meio da análise estatística multivariada, e com-

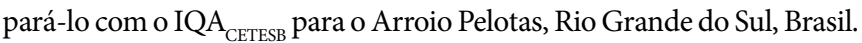

\section{MATERIAIS E MÉTODOS}

\section{Caracterização da área de estudo}

A Lei no 10.350/1994 do estado do Rio Grande do Sul (RIO GRANDE DO SUL, 1994), artigo 38, determina a existência de três regiões hidrográficas: região do Rio Uruguai, região Litorânea e região Guaíba. Nesse contexto, a área de estudo corresponde ao ponto de coleta GER 48

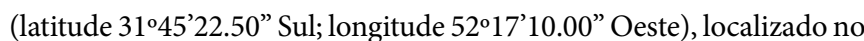
Arroio Pelotas (Figura 1), inserido na Bacia Hidrográfica Mirim-São Gonçalo, dentro da Região Hidrográfica da Bacia Litorânea (FEPAM, 2015). É importante destacar que no entorno do ponto amostral há diversas áreas antropizadas por processos de urbanização, conforme pode ser observado na Figura 1.

\section{Base de dados}

Foram utilizados os dados de qualidade da água disponibilizados pela FEPAM entre os anos de 1997 e 2013, considerando os seguintes parâmetros: oxigênio dissolvido (OD), coliformes termotolerantes (CT), potencial hidrogeniônico $(\mathrm{pH})$, demanda bioquímica de oxigênio $\left(\mathrm{DBO}_{5}\right)$, nitrogênio total $(\mathrm{NT})$, fósforo total $(\mathrm{PT})$, turbidez $(\mathrm{TH})$, sólidos totais $(\mathrm{ST})$ e temperatura da água $\left(\mathrm{T}_{\mathrm{H} 2 \mathrm{O}}\right)$. As coletas foram realizadas pela FEPAM de acordo com a metodologia descrita pela CETESB (1987), e os métodos analíticos, bem como a preservação das amostras, seguiram os procedimentos definidos pela Associação Americana de Saúde Pública (APHA, 2005).

\section{Análise estatística multivariada}

\section{Análise fatorial/análise de componentes principais}

A ACP foi escolhida para avaliar a relação entre as variáveis, evidenciando a participação individual dos elementos físicos, químicos e biológicos na qualidade da água do Arroio Pelotas.

Primeiramente, construiu-se uma matriz de dados expressos por $X=(x i, j)$, em que $i=1 \ldots n$ amostragens $(28)$ e $j=1 \ldots p$ variáveis (9). Em seguida, realizou-se a normalização dos dados no software Statistica, versão 7.0. Logo após, transformou-se a matriz de dados originais $(28 \times 9)$ em uma matriz de correlações [R] $(\mathrm{pxp})$, na qual $p$ corresponde às nove variáveis de qualidade da água a serem analisadas. Posteriormente, essa matriz foi decomposta e deu origem aos fatores. Esses fatores foram identificados e extraídos por intermédio da ACP, formando assim um novo conjunto de variáveis, denominadas de Componentes Principais (CP).

Segundo Hair et al. (2009) e Mingoti (2013), as três primeiras componentes devem explicar o máximo de variabilidade total dos dados, e a primeira não deve estar correlacionada com a segunda, nem a segunda com a terceira, nem a terceira com a primeira ou com a segunda, e assim sucessivamente, até que as CPs expliquem mais de $70 \%$ da variância total dos dados. Para a obtenção da matriz de correlação, bem como a análise fatorial (AF)/ACP, fez-se uso do software Statistica, versão 7.0.

Para validar a AF, aplicaram-se os testes de esfericidade de Bartlett e a medida de capacidade de adequação da amostra de Kaiser-Meyer-Olkin (KMO), mediante a função programada no software livre Action. Os testes de Bartlett e KMO testam a hipótese de que as variáveis não são correlacionadas na amostra. 
Após a obtenção da matriz de correlação, realizou-se o teste de hipóteses, com o objetivo de identificar se havia correlações significativas entre as variáveis, e a hipótese nula adotada foi a de que o coeficiente de correlação é 0 . Utilizou-se o teste $t$ de Student com nível de significância de 5\% (BARBETTA, 2012). O valor de $\mathrm{p}$ foi calculado com base na matriz de correlação pelo software Statistica, versão 7.0.

\section{Índice de qualidade da água modificado}

Após a realização da AF/ACP, procedeu-se ao cálculo do IQA ${ }_{\text {Mod }}$ obtido com base nos escores fatoriais. Segundo Costa et al. (2012), Moretto et al. (2012) e Sperling (2007), no cálculo do IQA apresentado pela NSF, o somatório dos pesos das variáveis deve ser igual a 1, e, quando necessário, deve-se realizar uma transformação matemática nos autovetores por meio da divisão de cada um dos valores pelo somatório total dos autovetores, obtendo-se assim os pesos calibrados.

A determinação do $\mathrm{IQA}_{\mathrm{Mod}}$ seguiu a metodologia utilizada pela CETESB (2016). Portanto, foram utilizados nove parâmetros de qualidade da água: $\mathrm{CT}, \mathrm{pH}, \mathrm{DBO}_{5}, \mathrm{NT}, \mathrm{PT}, \mathrm{TH}, \mathrm{T}_{\mathrm{H} 2 \mathrm{O}}$, ST e OD.
O IQA é calculado segundo a Equação 1, e os valores do índice variam de 0 a 100.

$\mathrm{IQA}=\prod_{1}^{\mathrm{n}} \mathrm{qi}^{\mathrm{wi}}$

Em que:

IQA = índice de qualidade da água, entre 0 e 100;

qi $=$ qualidade do i-ésimo parâmetro, entre 0 e 100, obtido da respectiva curva média de variação de qualidade, em função de sua concentração ou medida; wi = peso correspondente ao i-ésimo parâmetro, entre 0 e 1 , atribuído em função da sua importância para a conformação global de qualidade, obtido através da AF e extraído através da ACP;

$\mathrm{n}$ = número de variáveis que entram no cálculo do IQA.

O resultado do IQA foi classificado em cinco faixas de acordo com as faixas estabelecidas pela CETESB (2016) e aplicadas no estado do Rio Grande do Sul, como se observa na Tabela 1.

Após o cálculo do $\mathrm{IQA}_{\mathrm{Mod}}$, realizou-se a sua comparação com o IQA $_{\text {CETESB }}$.

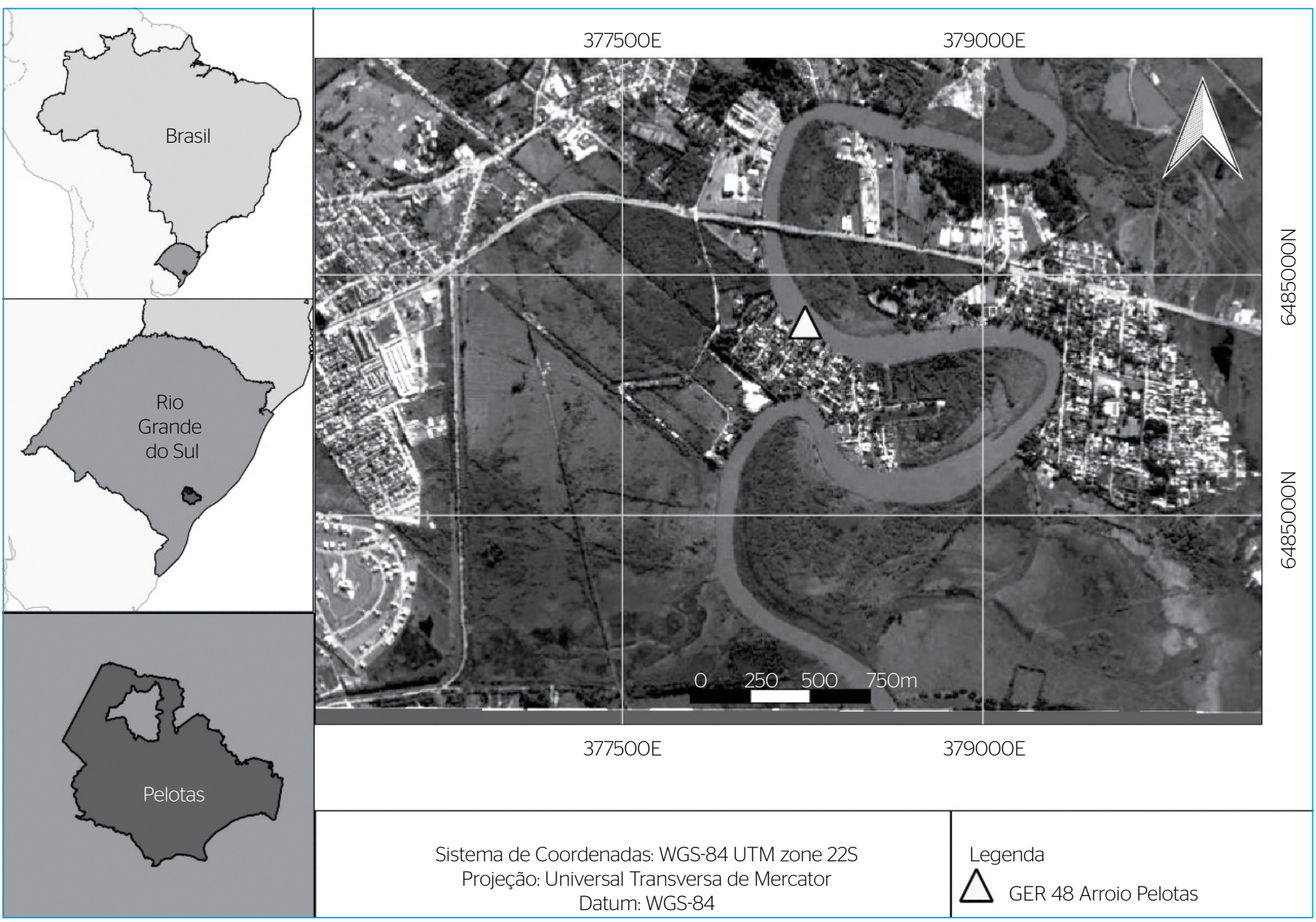

Figura 1 - Mapa de localização do ponto GER 48 no Arroio Pelotas, Rio Grande do Sul, Brasil. 


\section{RESULTADOS E DISCUSSÃO}

Na Tabela 2 estão apresentados os resultados encontrados para a validação da AF por meio do teste de esfericidade de Bartlett e adequacidade de KMO.

Como o valor de $\mathrm{p}<0,05$ e $\mathrm{KMO}>0,5$, pode-se afirmar que a proposição da AF é adequada para a matriz de correlação dos dados.

Segundo Hair et al. (2009) e Corrar, Paulo e Dias Filho (2014), os coeficientes de correlação superiores a 0,7 expressam forte relação entre as variáveis de qualidade de água. Assim, as variáveis CT, PT, NT, OD, $\mathrm{pH} \mathrm{e}_{\mathrm{H} 2 \mathrm{O}}$ apresentaram maiores coeficientes de correlação com maior número de variáveis, como pode ser observado na Tabela 3.

Para verificar as correlações mais significativas entre as variáveis na matriz de correlação, analisou-se o valor de p. De acordo com a Tabela 3, as variáveis PT e $\mathrm{T}_{\mathrm{H} 2 \mathrm{O}}$ e $\mathrm{OD}$ e CT apresentaram valor de p menor que o nível de significância adotado. Portanto, rejeitou-se a hipótese nula, concluindo que efetivamente existe relação significativa apenas entre essas variáveis.

Sendo assim, se ocorrer aumento na $\mathrm{T}_{\mathrm{H} 2 \mathrm{O}}$, haverá decréscimo na concentração de PT. Isso pode ser explicado pelo fato de a elevação da temperatura facilitar a taxa de crescimento microbiano e as reações químicas, físicas e biológicas, diminuindo a concentração de PT, pois este é um nutriente indispensável para o metabolismo microbiano (SPERLING, 2005), enquanto a correlação significativa negativa entre OD e CT pode ser justificada pelo processo de respiração biológica, que reduz as concentrações de OD (HELLER \& PÁDUA, 2010).

A Tabela 4 apresenta os componentes principais identificados seguindo o critério de Kaiser (1958), Jolliffe (2002) e Hair et al. (2009).

Podemos observar que pelo primeiro fator gerado foi possível explicar $70,63 \%$ da variância total dos dados. Portanto, os coeficientes

Tabela 1 - Classificação do índice de qualidade da água modificado $\left(\mathrm{IQA} \mathrm{A}_{\mathrm{mod}}\right)$, de acordo com as faixas estabelecidas pela Companhia Ambiental do Estado de São Paulo.

\begin{tabular}{l|c|c} 
Classificação & Cor & Faixa de IQA \\
\hline Ótima & & $90<1 \mathrm{Q} \leq 100$ \\
\hline Boa & & $70<1 \mathrm{QA} \leq 90$ \\
\hline Aceitável & & $50<1 \mathrm{Q} A \leq 70$ \\
\hline Ruim & & $25<1 \mathrm{Q} A \leq 50$ \\
\hline Péssima & & $0<1 \mathrm{Q} A \leq 25$ \\
\hline
\end{tabular}

IQA: índice de qualidade da água.

Tabela 2 - Validação da análise fatorial por meio dos testes de esfericidade e capacidade de adequação da amostra.

\begin{tabular}{l|c}
$\begin{array}{l}\text { Teste de esfericidade } \\
\text { de Bartlett }\end{array}$ & $\begin{array}{c}\text { Medida de capacidade de adequação da } \\
\text { amostra de Kaiser-Meyer-Olkin (KMO) }\end{array}$ \\
\hline $\mathrm{p}$ & $\mathrm{KMO}$ \\
\hline $5,63.10^{-280}$ & 0,9179 \\
\hline
\end{tabular}

de cada variável, do primeiro escore fatorial extraído, compuseram o $\mathrm{IQA}_{\text {Mod }}$, conforme observamos na Tabela 5.

Vê-se na Tabela 5 que os escores fatoriais foram calibrados com o intuito de apresentar o somatório igual a uma unidade, da mesma forma como foi apontado o somatório dos pesos da CETESB. Os pesos obtidos para o IQA ${ }_{\text {Mod }}$ foram inferiores aos utilizados pela CETESB em seis variáveis de qualidade da água (CT, PT, NT, OD, $\mathrm{pH}_{\text {e }} \mathrm{T}_{\mathrm{H} 2 \mathrm{O}}$ ) e em três variáveis foram superiores $\left(\mathrm{DBO}_{5}, \mathrm{ST}\right.$ e $\left.\mathrm{TH}\right)$. Logo, as variáveis mais importantes para avaliar a qualidade da água do Arroio Pelotas

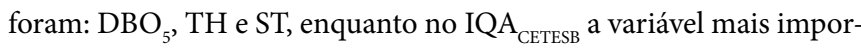
tante foi o $\mathrm{OD}$, seguida pelas variáveis $\mathrm{CT}$ e $\mathrm{pH}$.

Para Libânio (2010), que trabalhou com estudos de monitoramento ambiental na Bacia Hidrográfica do Rio Pardo, Rio Grande do Sul, calibrando o IQA originalmente desenvolvido pela NSF $\left(\mathrm{IQA}_{\mathrm{NSF}}\right)$

Tabela 3 - Matriz de correlação e valores de $\mathrm{p}$ para os dados de qualidade da água do Arroio Pelotas, RS, Brasil.

\begin{tabular}{|c|c|c|c|c|c|c|c|c|c|c|}
\hline & & CT & $\mathrm{DBO}_{5}$ & PT & NT & OD & $\mathrm{pH}$ & ST & $\mathrm{T}_{\mathrm{H} 2 \mathrm{O}}$ & TH \\
\hline & $r$ & 1,00 & & & & & & & & \\
\hline & p & - & & & & & & & & \\
\hline & $r$ & 0,29 & 1,00 & & & & & & & \\
\hline 20 & $p$ & 0,81 & - & & & & & & & \\
\hline & $r$ & 0,95 & $-0,02$ & 1,00 & & & & & & \\
\hline & p & 0,19 & 0,98 & - & & & & & & \\
\hline$N T$ & $r$ & 0,43 & $-0,73$ & 0,69 & $1, \mathrm{OO}$ & & & & & \\
\hline & p & 0,71 & 0,47 & 0,50 & - & & & & & \\
\hline & $r$ & $-0,99$ & 0,39 & 0,91 & 0,33 & 1,00 & & & & \\
\hline & $p$ & 0,03 & 0,74 & 0,27 & 0,78 & - & & & & \\
\hline & $r$ & 0,85 & $-0,24$ & 0,97 & 0,83 & 0,79 & 1,00 & & & \\
\hline דו & p & 0,34 & 0,84 & 0,14 & 0,37 & 0,41 & - & & & \\
\hline$C T$ & r & $-0,17$ & 0,88 & $-0,47$ & $-0,96$ & $-0,06$ & $-0,65$ & 1,00 & & \\
\hline & p & 0,88 & 0,30 & 0,68 & 0,17 & 0,95 & 0,54 & - & & \\
\hline & $r$ & $-0,94$ & 0,03 & $-0,99$ & $-0,70$ & $-0,90$ & $-0,97$ & 0,48 & 1,00 & \\
\hline${ }^{1} \mathrm{H} 2 \mathrm{O}$ & $p$ & 0,21 & 0,98 & 0,01 & 0,50 & 0,28 & 0,13 & 0,67 & - & \\
\hline TH & $r$ & $-0,85$ & $-0,74$ & $-0,65$ & 0,09 & $-0,91$ & $-0,46$ & $-0,35$ & 0,64 & 1,00 \\
\hline & p & 0,35 & 0,46 & 0,54 & 0,94 & 0,27 & 0,69 & 0,77 & 0,55 & - \\
\hline
\end{tabular}

$\mathrm{CT}$ : coliformes termotolerantes; $\mathrm{DBO}_{5}$ : demanda bioquímica de oxigênio; PT: fósforo total; NT: nitrogênio total; OD: oxigênio dissolvido; pH: potencial hidrogeniônico ST: sólidos totais; $T_{\text {H2O }}$ : temperatura da água; TH: turbidez; r: coeficiente de correlação; $\mathrm{p}$ : valor de $\mathrm{p}$

Tabela 4 - Variância total da amostra de qualidade da água do Arroio Pelotas, RS, Brasil.

\begin{tabular}{l|c|c} 
Fator & $\begin{array}{c}\text { Variância total } \\
\text { explicada (\%) }\end{array}$ & $\begin{array}{c}\text { Variância total } \\
\text { acumulada (\%) }\end{array}$ \\
\hline 1 & 70,63 & 70,63 \\
\hline 2 & 29,37 & 100,00 \\
\hline
\end{tabular}


Tabela 5 - Escores fatoriais e os pesos calibrados para compor o índice de qualidade da água modificado.

\begin{tabular}{l|c|c|c} 
Variáveis & Escore fatorial 1 & Peso calibrado & Pesos CETESB \\
\hline $\mathrm{CT}$ & 0,12 & 0,09 & 0,15 \\
\hline $\mathrm{DBO}_{5}$ & 0,22 & 0,16 & 0,10 \\
\hline $\mathrm{PT}$ & 0,13 & 0,09 & 0,10 \\
\hline $\mathrm{NT}$ & 0,12 & 0,09 & 0,10 \\
\hline $\mathrm{OD}$ & 0,14 & 0,11 & 0,17 \\
\hline $\mathrm{pH}$ & 0,13 & 0,09 & 0,12 \\
\hline $\mathrm{ST}$ & 0,17 & 0,12 & 0,08 \\
\hline $\mathrm{T}_{\mathrm{H} 2 \mathrm{O}}$ & 0,13 & 0,09 & 0,10 \\
\hline $\mathrm{TH}$ & 0,21 & 0,15 & 0,08 \\
\hline$\Sigma$ & 1,38 & 1,00 & 1,00 \\
\hline
\end{tabular}

CETESB: Companhia Ambiental do Estado de São Paulo; CT: coliformes termotolerantes; $\mathrm{DBO}_{5}$ : demanda bioquímica de oxigênio; PT: fósforo total; NT: nitrogênio total; OD: oxigênio dissolvido; pH: potencial hidrogeniônico; ST: sólidos totais; $T_{\text {H22: }}$ temperatura da água; $T H$ : turbidez.

com base na Resolução no 357/2005 do Conselho Nacional do Meio Ambiente (CONAMA), os principais parâmetros incluídos no cálculo do IQA para a Bacia Hidrográfica do Rio Pardo, em ordem decrescente de importância, foram: CT, OD e PT. Para justificar a modificação do $\mathrm{IQA}_{\mathrm{NSP}}$, os autores ressaltam ainda que em muitos casos esse índice não representa a realidade dos mananciais superficiais existentes no Brasil.

Estudos realizados por Pinto et al. (2009) e Toledo e Nicolella (2002) também verificaram diferentes pesos das variáveis de qualidade da água a serem utilizadas no cálculo do IQA, obtendo IQAs específicos para as áreas de estudo. Sendo assim, pode-se destacar a importância da readequação dos pesos em IQA para cada manancial, com o intuito de obter as variáveis mais importantes e seus respectivos pesos, representando as reais características de cada local.

Na Tabela 6 está apresentada a variação temporal da qualidade de água observada no ponto de monitoramento no Arroio Pelotas, estimada em função do IQA Mod $_{\text {e do IQA }}$ CETESB.

Observa-se na Tabela 6 que nos anos 2005, 2006 e 2013 ocorreu uma mudança da faixa de qualidade da água quando comparamos a aplicação dos IQAs, passando de aceitável para boa. Essa mudança deve-se à maior sensibilidade do $\mathrm{IQA}_{\mathrm{Mod}}$, decorrente da redistribuição de seus
Tabela 6 - Variação temporal do comportamento do índice de qualidade da água modificado (IQA ${ }_{\text {mod }}$ e do índice de qualidade da água utilizado pela Companhia Ambiental do Estado de São Paulo $\left(\mathrm{IQA}_{\mathrm{CETESB}}\right.$ ) no Arroio Pelotas em função das faixas de qualidade da água estabelecidas para o Rio Grande do Sul.

\begin{tabular}{l|c|c|c|c} 
Ano & IQA $_{\text {Mod }}$ & Classificação & IQA $_{\text {CETESB }}$ & Classificação \\
\hline 1997 & 29,95 & Péssima & 27,03 & Péssima \\
\hline 1998 & 10,23 & Péssima & 14,12 & Péssima \\
\hline 1999 & 10,40 & Péssima & 14,31 & Péssima \\
\hline 2000 & 10,96 & Péssima & 15,57 & Péssima \\
\hline 2001 & 10,47 & Péssima & 14,50 & Péssima \\
\hline 2002 & 10,70 & Péssima & 15,00 & Péssima \\
\hline 2003 & 6,16 & Péssima & 6,01 & Péssima \\
\hline 2005 & 43,78 & Ruim & 45,10 & Ruim \\
\hline 2006 & 66,86 & Aceitável & 74,12 & Boa \\
\hline 2007 & 12,99 & Péssima & 10,60 & Péssima \\
\hline 2008 & 19,56 & Péssima & 11,49 & Péssima \\
\hline 2009 & 47,26 & Ruim & 38,97 & Ruim \\
\hline 2010 & 45,48 & Ruim & 46,79 & Ruim \\
\hline 2011 & 42,09 & Ruim & 34,01 & Ruim \\
\hline 2012 & 66,51 & Aceitável & 69,74 & Aceitável \\
\hline 2013 & 68,40 & Aceitável & 76,63 & Boa \\
\hline
\end{tabular}

pesos. Nos anos supracitados, as variáveis $\mathrm{TH}, \mathrm{DBO}_{5}$ e ST apresentaram concentrações mais elevadas, quando comparadas com as concentrações no restante da série histórica, o que fez com que sua nota $\left(\mathrm{q}_{\mathrm{i}}\right)$ fosse mais baixa e, em decorrência disso, apresentasse qualidade da água menor. Esses resultados vêm chancelar a importância da calibração de IQAs para ecossistemas específicos.

\section{CONCLUSÃO}

Com base nos resultados encontrados, conclui-se que, ao aplicar a AF/ACP nos dados de qualidade da água do Arroio Pelotas, foram obtidos pesos diferentes dos utilizados pela CETESB para todas as variáveis que compõem o cálculo do IQA, demonstrando assim a necessidade da readequação desse índice para esse ecossistema específico.

\section{REFERÊNCIAS}

ABREU, C.H.M.; CUNHA, A.C. (2015) Qualidade da Água em Ecossistemas Aquáticos Tropicais Sob Impactos Ambientais no Baixo Rio Jari-AP: Revisão Descritiva. Biota Amazônia, v. 5, n. 2, p. 119-131. http:// dx.doi.org/10.18561/2179-5746/biotaamazonia.v5n2p119-131
AL-MUTAIRI, N.; ABAHUSSAIN, A.; EL-BATTAY, A. (2014) Spatial and temporal characterizations of water quality in Kuwait Bay. Marine Pollution Bulletin, v. 83, n. 1, p. 127-131. https://doi.org/10.1016/j. marpolbul.2014.04.009 
AMERICAN PUBLIC HEALTH ASSOCIATION (APHA). (2005) Standard methods for the examination of water and wastewater. 21. ed. Washington, D.C.: APHA/AWWA/WEF.

BARBETTA, P.A. (2012) Estatística Aplicada às Ciências Sociais. 8. ed. Florianópolis: Editora da UFSC. 315 p.

BERTOSSI, A.P.A.; CECILIO, R.A.; NEVES, M.A.; GARCIA, G.O. (2O13) Qualidade da água em microbacias hidrográficas com diferentes coberturas do solo no sul do Espírito Santo. Revista Arvore, v. 37 , n. 1, p. 107-117. http://dx.doi.org/10.1590/S0100-67622013000100012

BLUME, K.K; MACEDO, J.C; MENEGUZZI, A.; SILVA, L.B; QUEVEDO, D.M; RODRIGUES, M.A.S. (2010) Water quality assessment of the Sinos River, Southern Brazil. Brazilian Journal of Biology, v. 70, n. 4, p. 1185-1193. http://dx.doi.org/10.1590/S1519-69842010000600008

COMPANHIA AMBIENTAL DO ESTADO DE SÃO PAULO (CETESB). (1987) Guia de coleta e preservação de amostras de água. São Paulo: CETESB.

. (2016) Indices de qualidade das águas. São Paulo: CETESB.

CORRAR, J.L.; PAULO, E.; DIAS FILHO, M.J. (2014) Análise Multivariada: para os Cursos de Administração, Ciências Contábeis e Economia. São Paulo: Atlas. 586 p.

COSTA, A.B.; POSSELT, E.L.; MENEZES, C.M.; LOBO, E.A. (2012) Desenvolvimento e aplicação de índices de qualidade da água. Caderno de Pesquisa, v. 24, n. 1, p. 67-78. http://dx.doi.org/10.17058/cp.v24i1.4398

CUNHA, R.W.; GARCIA JR., M.D.N.; ALBERTONI, E.F.; SILVA, C.P. (2O13) Qualidade de água de uma lagoa rasa em meio rural no sul do Brasil. Revista Brasileira Engenharia Agrícola Ambiental, v. 17, n. 7 , p. 770-779. http://dx.doi.org/10.1590/S1415-43662013000700012

FUNDAÇÃO ESTADUAL DE PROTEÇÃO AMBIENTAL LUIS HENRIQUE ROESSLER (FEPAM). (2015) Monitoramento da Qualidade da Agua da Região Hidrográfica das Bacias Litorâneas. Disponível em: <http:/l www.fepam.rs.gov.br/>. Acesso em: 10 mar. 2016.

GOMES, A.I.; PIRES, J.C.M.; FIGUEIREDO, S.A.; BOAVENTURA, R.A.R. (2014) Optimization of River Water Quality Surveys by Multivariate Analysis of Physicochemical, Bacteriological and Ecotoxicological Data. Water Resources Management, v. 28, n. 5, p. 1345-1361. https:// doi.org/10.1007/s11269-014-0547-9

HAIR, J.; BLACK, W.; BABIN, B.; ANDERSON, R.; TATHAM, R. (2009) Análise Multivariada de Dados. 6. ed. Porto Alegre: Bookman.

HELLER, L; PADUA, L. (2010) Abastecimento de Agua para Consumo Humano. 2. ed. Belo Horizonte: Editora da UFMG. v. 1.

JOLLIFFE, I.T. (2002) Principal Component Analysis. 2. ed. Nova York: Springer. $518 \mathrm{p}$

KAISER, H.F. (1958) The varimax criteria for analitical rotation in fator analysis. Psychometrica, v. 23, p. 187-200. https://doi.org/10.1007/ BFO2289233

LIBÂNIO, M. (2010) Fundamentos de Qualidade e Tratamento de Agua. Campinas: Átomo.
MINGOTI, S.A. (2013) Análise de dados através de métodos de estatística multivariada: uma abordagem aplicada. 2. ed. Minas Gerais: Editora da UFMG. 295 p.

MORETTO, D.L;; PANTA, R.E.; COSTA, A.B.; LOBO, E.A. (2012) Calibration of water quality index (WQI) based on Resolution no $357 / 2005$ of the Environment National Council (CONAMA). Acta Limnologica Brasiliensia, Rio Claro, v. 24, n. 1, p. 29-42. http://dx.doi. org/10.1590/S2179-975X2012005000024

OLSEN, R.L.; CHAPPELL, R.W:; LOFTIS, J.C. (2012) Water quality sample collection, data treatment and results presentation for principal components analysis - literature review and Illinois River watershed case study. Water Research, v. 46, n. 9, p. 3110-3122. https://doi.org/10.1016/j.watres.2012.03.028

OUYANG, Y. (2005) Evaluation of river water quality monitoring stations by principal component analysis. Water Research, v. 39, n. 12, p. 2621-2635. https://doi.org/10.1016/|.watres.2005.04.024

PINTO, D.B.F; SILVA, A.M.; MELLO, C.R.; COELHO, G. (2009) Qualidade da água do Ribeirão Lavrinha na região Alto Rio Grande - MG, Brasil. Ciência e Agrotecnologia. http://dx.doi.org/10.1590/ S1413-70542009000400028

POLETO, C. (2014) Bacias Hidrográficas e Recursos Hídricos. Rio de Janeiro: Interciência. 272 p.

RIO GRANDEDOSUL.(1994) Lei no 10.350, de3O de dezembro de1994. Institui O Sistema Estadual de Recursos Hídricos, regulamentando o Artigo 171 da Constituição do Estado do Rio Grande do Sul. Diário Oficial do Estado do Rio Grande do Sul, Porto Alegre.

SILVEIRA, T; REGO, N.A.C.; SANTOS, J.W.B.; ARAÚJO, M.S.B. (2014) Qualidade da Agua e Vulnerabilidade dos Recursos Hídricos Superficiais na Definição das Fragilidades Potencial e Ambiental de Bacias Hidrográficas. Revista Brasileira de Geografia Física, v. 7. n. 4, p. 643-652.

SIMEONOV, V;; STRATIS, J.A.; SAMARA, C.; ZACHARIADIS, G.; VOUTSA, D.; ANTHEMIDIS, A.; SOFONIOU, M.; KOUIMTZIS, T. (2003) Assessment of the surface water quality in northern Greece. Water Research, v. 37, p. 4119-4124. https://doi.org/10.1016/S00431354(O3)00398-1

SPERLING, M.V. (2005) Introdução à qualidade das águas e ao tratamento de esgotos. 3. ed. Belo Horizonte: DESA/UFMG. 452 p. (Coleção Princípios do Tratamento Biológico de Águas Residuárias, v. 1).

(2007) Estudos e modelagem da qualidade da água de rios. Belo Horizonte: DESA/UFMG. 588 p. (Coleção Princípios do Tratamento Biológico de Águas Residuárias, v. 7).

SUN, W.; XIA, C.; XU, M.; GUO, J.; SUN, G. (2016) Application of modified water quality indices as indicators to assess the spatial and temporal trends of water quality in the Dongjiang River. Ecological Indicators, v. 66, p. 306-312. http://dx.doi.org/10.1016/j. ecolind.2016.01.054

TOLEDO, L.G.; NICOLELLA, G. (2002) Indice de Qualidade de Água em microbacia sob uso agrícola e urbano. Scientia Agricola, v. 59, n.1, p. 181-186. http://dx.doi.org/10.1590/S0103-90162002000100026 